\title{
ISOTOPY AND IDENTITIES IN ALTERNATIVE ALGEBRAS
}

\author{
M. BABIKOV
}

(Communicated by Lance W. Small)

\begin{abstract}
In this paper we show how to construct an isomorphism between an alternative algebra $A$ over a field of characteristic $\neq 3$ and its isotope $A^{(1+c)}$, where $c$ is an element of Zhevlakov's radical of $A$. This leads to the equivalence of any polynomial identity $f=0$ in alternative algebras and the isotope identity $f^{(s)}=0$.
\end{abstract}

Given an invertible element $s$ of an alternative algebra $A$, we can form a new algebra by taking the same linear structure but a new multiplication

$$
x *_{s} y=(x s) y .
$$

The resulting algebra, denoted $A^{(s)}$, is also alternative (see [1]) and is called an $s$-isotope. Associative and Cayley isotopes are always isomorphic [2], and an isotope of a finite-dimensional alternative algebra over an algebraically closed field of characteristic $\neq 3$ is isomorphic to the original algebra as well [1]. We consider the case of an arbitrary alternative algebra over a field of characteristic $\neq 3$ with nonzero Zhevlakov radical, and a particular choice of $s$, and construct an explicit isomorphism between $A$ and $A^{(s)}$. As a consequence, we derive the equivalence of any polynomial identity and its isotope in an arbitrary alternative algebras over a field of characteristic $\neq 3$. The paper has benefited from many discussions with J. Ferrar, and I would like to thank him for his valuable help and encouragement.

Theorem 1. There is a set of coefficients $t_{j}^{i}, i, j \geq 0$, such that $t_{0}^{0}=1$ and for any integer $m$, any alternative algebra $A$ over a field of characteristic $\neq 3$ and any elements $a, b, c$ from $A$ such that $\operatorname{Id}_{A}(c)^{m}=0$, the polynomial $t(x)=\sum_{i, j} t_{j}^{i} c^{i} x c^{j}$ satisfies

$$
t(a) *_{1+c} t(b)=t(a b) .
$$

Proof. We will show the way to calculate the coefficients $t_{j}^{i}$ such that (1) holds for any alternative algebra $A$. Clearly,

$$
\begin{aligned}
t(a) *_{1+c} t(b) & =t(a) t(b)+(t(a) c) t(b) \\
& =\sum t_{j}^{i} t_{l}^{k}\left(c^{i} a c^{j}\right)\left(c^{k} b c^{l}\right)+\sum t_{j}^{i} t_{l}^{k}\left(c^{i} a c^{j+1}\right)\left(c^{k} b c^{l}\right) \\
& =\sum t_{l}^{k}\left(t_{j}^{i}+t_{j-1}^{i}\right)\left(c^{i} a c^{j}\right)\left(c^{k} b c^{l}\right),
\end{aligned}
$$

Received by the editors March 28, 1995.

1991 Mathematics Subject Classification. Primary 17D05.

(C) 1997 American Mathematical Society 
where $t_{-1}^{i}=0$ and $0 \leq i, j, k, l \leq m-1$. For brevity we shall denote $t_{l}^{k}\left(t_{j}^{i}+t_{j-1}^{i}\right)$ simply by $t_{l j}^{k i}$; then

$$
\begin{gathered}
t(a) *_{1+c} t(b)-t(a b)=\sum t_{l j}^{k i}\left(\left(c^{i} a c^{j+k}\right)\left(b c^{l}\right)+\left(c^{i} a c^{j}, c^{k}, b c^{l}\right)\right) \\
-\sum t_{l}^{i}\left(\left(c^{i} a\right)\left(b c^{l}\right)-\left(c^{i}, a, b\right) c^{l}+\left(c^{i} a, b, c^{l}\right)\right) \\
=\sum_{i, l, n} \sum_{j+k=n} t_{l j}^{k i}\left(c^{i} a c^{n}\right)\left(b c^{l}\right)+\sum t_{l j}^{k i} c^{j+l}\left(a, b, c^{k}\right) c^{i} \\
\quad-\sum t_{l}^{i}\left(c^{i} a\right)\left(b c^{l}\right)-\sum t_{l}^{i}\left(c^{i} a, b, c^{l}\right)+\sum t_{l}^{i}\left(a, b, c^{i}\right) c^{l} .
\end{gathered}
$$

We need (1) to be true for any alternative algebra, in particular for an associative one, for which all the associators in (2) are zeroes and (1) is equivalent to

$$
\sum_{i, l, n} \sum_{j+k=n} t_{l j}^{k i} c^{i} a c^{n} b c^{l}-\sum t_{l}^{i} c^{i} a b c^{l}=0 .
$$

For the cases $n=0$ and $n \geq 1$ we get that

$$
\begin{gathered}
t_{l 0}^{0 i}=t_{l}^{i}, \\
\sum_{j+k=n} t_{l j}^{k i}=0,
\end{gathered}
$$

for any $i$ and $l$. It is easy to see that (4) yields

$$
t_{l}^{i}=t_{0}^{i} \cdot t_{l}^{0}
$$

But then

$$
\sum_{j+k=n} t_{l j}^{k i}=t_{l}^{i} \sum_{j+k=n}\left(t_{j}^{k}+t_{j-1}^{k}\right),
$$

and therefore (5) yields

$$
\sum_{j+k=n} t_{j}^{k}=-\sum_{j+k=n-1} t_{j}^{k}
$$

Consequently, since $\sum_{j+k=1} t_{j}^{k}=t_{0}^{0}=1$ and by induction on $n$,

$$
\sum_{j+k=n} t_{j}^{k}=(-1)^{n}
$$

Now we assume (6) and (7), which implies (3). Going back to the alternative case we note that (4) and (5) also imply

$$
\sum_{i, l, n} \sum_{j+k=n} t_{l j}^{k i}\left(c^{i} a c^{n}\right)\left(b c^{l}\right)-\sum t_{l}^{i}\left(c^{i} a\right)\left(b c^{l}\right)=0 .
$$

So in the expression (2) only sums with the associators are left. It is easy to see that in any alternative algebra the Moufang identities imply

$$
\begin{aligned}
\left(a, b, c^{k+l}\right) & =-\left(a, c^{l}, c^{k} b\right)+b\left(a, c^{l}, c^{k}\right)+c^{l}\left(a, b, c^{k}\right) \\
& =\left(a, b, c^{l}\right) c^{k}+c^{l}\left(a, b, c^{k}\right) .
\end{aligned}
$$


Using this identity we get

$$
\begin{aligned}
t(a) * t(b)-t(a b)= & \sum t_{l j}^{k i}\left(-\left(a, b, c^{l+j}\right) c^{k+i}+\left(a, b, c^{l+j+k}\right) c^{i}\right) \\
& -\sum t_{l}^{i}\left(a, b, c^{l}\right) c^{i}+\sum t_{l}^{i}\left(a, b, c^{i}\right) c^{l} \\
= & \sum T_{p q}\left(a, b, c^{p}\right) c^{q}
\end{aligned}
$$

where

$$
T_{p q}=t_{q}^{p}-t_{p}^{q}-\sum_{l+j=p, k+i=q} t_{l j}^{k i}+\sum_{l+j+k=p,(i=q)} t_{l j}^{k q} .
$$

Let us simplify this expression for $T_{p q}$. Clearly by (7)

$$
\begin{aligned}
\sum_{l+j+k=p} t_{l j}^{k q} & =\sum_{k+l=p-j} t_{l}^{k} \sum_{j} t_{0 g}^{0 j}=\sum_{j}(-1)^{p-j} t_{0 g}^{0 j} \\
& =\sum_{j}(-1)^{p-j}\left(t_{j}^{q}+t_{j-1}^{q}\right)=t_{p}^{q},
\end{aligned}
$$

and so

$$
T_{p q}=t_{q}^{p}-\sum_{l+j=p, k+i=q} t_{l j}^{k i}
$$

Now we use induction on $d=p+q$ to prove the existence of $t_{0}^{d}$ and $t_{d}^{0}$ such that $T_{p q}=0$ for any $p+q \leq d$. Note that although we do it for any $p$ and $q$, we need $T_{p q}=0$ only for $p \neq 0$, because otherwise in (8) we have $\left(a, b, c^{p}\right) c^{q}=0$. First, we consider the case $q=0$ and find $t_{0}^{d}$ :

$$
t_{0}^{d}+t_{d}^{0}=\sum_{i+j=d} t_{j}^{i}-\sum_{i+j=d, i \neq d, j \neq d} t_{j}^{i}=(-1)^{d}-\sum_{i+j=d, i \neq d, j \neq d} t_{j}^{0} t_{0}^{i} .
$$

So, since $T_{p q}=0$,

$$
\begin{aligned}
t_{0}^{d} & =\sum_{l+j=d} t_{l}^{0}\left(t_{j}^{0}+t_{j-1}^{0}\right)=\sum_{l+j=d, l \neq d, j \neq d} t_{l}^{0}\left(t_{j}^{0}+t_{j-1}^{0}\right)+t_{d-1}^{0}+2 t_{d}^{0} \\
& =\sum_{l+j=d, l \neq d, j \neq d} t_{l j}^{00}+t_{d-1}^{0} 2\left((-1)^{d}-\sum_{i+j=d, i \neq d, j \neq d} t_{j}^{0} t_{0}^{i}-t_{0}^{d}\right),
\end{aligned}
$$

and we get

$$
t_{0}^{d}=\frac{1}{3}\left(\sum_{l+j=d, l \neq d, j \neq d} t_{l j}^{00}+t_{d-1}^{0}+2\left((-1)^{d}-\sum_{i+j=d, i \neq d, j \neq d} t_{j} t^{i}\right)\right) .
$$

To complete the proof we must show that $T_{q p}=0$ for any $p, q$ such that $p+q=d$ based on the assumption that this is true for $p+q \leq d-1$. If $p \neq 0$ and $q \neq 0$, then

$$
T_{p q}=t_{q}^{p}-\sum_{k+i=q} t_{00}^{k i} \sum_{l+j=p} t_{l j}^{00}=t_{q}^{p}-t_{0}^{p} t_{q}^{0}=0 .
$$


The case $q=0$ follows from (9) and for $p=0$ we have

$$
\begin{aligned}
T_{0 d} & =t_{d}^{0}-\sum_{k+i=d} t_{0}^{k} t_{0}^{i}=t_{d}^{0}-\sum_{k+i=d}\left(\sum_{l+j=k} t_{l j}^{00}\right) t_{0}^{i} \\
& =t_{d}^{0}-\sum_{j \leq d} t_{0 j}^{00} \sum_{i+l=d-j} t_{l}^{i}=t_{d}^{0}-\sum_{j \leq d}\left(t_{j}^{0}+t_{j-1}^{0}\right)(-1)^{d-j} \\
& =t_{d}^{0}-\left(\sum_{j \leq d} t_{j}^{0}(-1)^{d-j}-\sum_{j<d}\left(t_{j}^{0}\right)(-1)^{d-j}\right)=0 .
\end{aligned}
$$

This proves the theorem.

Corollary 1. Let $A, c$ and $t(x)$ be the same as in the Theorem 1; then the mapping $t: x \rightarrow t(x)$ is an isomorphism of $A$ onto $A^{(1+c)}$.

Proof. Theorem 1 states that $t$ is a homomorphism. To prove that $t$ is surjective, we have to find a polynomial $T=t^{-1}$ such that $t(T(a))=a$. Let us use induction on $m=\operatorname{deg}(c)$. For $m=1$ the statement is obvious: $T=1$. Assume that $T_{1}$ is the required polynomial for the case of $\operatorname{deg}(c)=m-1$; then for $\operatorname{deg}(c)=m$ we have $t\left(T_{1}(a)\right)=a+R(a)$, where $R$ is a homogeneous polynomial linear in $a$ and of degree $m-1$ in $c$. We set

$$
T(a)=T_{1}(a)-T_{1}(R(a)) .
$$

Then $R(R(a))=0$, since $R(R(a))$ is of degree $2(m-1) \geq m$ in $c$, and we have

$$
t(T(a))=a+R(a)-R(a)-R(R(a))=a-R(R(a))=a .
$$

This completes the proof.

Let $x_{1}, \ldots, x_{k}$ be some generators of a free alternative algebra $A_{0}$, and $f$ a polynomial in $x_{1}, \ldots, x_{k}$. Since $A_{0}$ is free, for any $s \in A_{0}$ there is a homomorphism $\phi_{s}: A_{0} \rightarrow A_{0}^{(s)}$ such that $x_{i} \mapsto x_{i}$. We denote the image of $f$ by $f^{(s)}$.

Theorem 2. Any alternative algebra $A$ over a field of characteristics $\neq 3$ with a polynomial identity $f=0$ satisfies also $f^{(s)}=0$ for any $s \in A$.

Proof. The variety of alternative algebras is homogeneous ([3], page 8); therefore we need to consider only the case of homogeneous polynomial $f$. Let $A_{0}$ be a free alternative algebra on $k+1$ generators $x_{1}, \ldots, x_{k}, c$. By Theorem 1 and the corollary,

$$
f^{(1+c)}\left(x_{1}, \ldots, x_{k}\right)=t\left(f\left(t^{-1} x_{1}, \ldots, t^{-1} x_{k}\right)\right)+c_{m},
$$

where $c_{m} \in \operatorname{Id}(c)^{m}$ and $m=\operatorname{deg}(f)$. Consider the homogeneous component of degree $m-1$ in $c$ :

$$
f^{(c)}\left(x_{1}, \ldots, x_{k}\right)=\Delta_{c}^{m-1} t\left(f\left(t^{-1} x_{1}, \ldots, t^{-1} x_{k}\right)\right) \in \operatorname{Id}(f) .
$$

Here $\Delta_{c}^{m-1}$ is the linearization operator (see [3]), and $\operatorname{Id}(f)$ denotes the ideal generated by all values of $f$. Since $A_{0}$ is free and $x_{1}, \ldots, x_{k}, c$ are its generators, (10) holds for any algebra $A$ and for any elements $x_{1}, \ldots, x_{k}, c$ in $A$. This proves the theorem. 
For algebras over a field of characteristics 3 Theorem 2 is false. Consider a commutative but not associative alternative algebra; it satisfies $[x, y]=0$. On the other hand,

$$
[x, y]^{(c)}=(x c) y-(y c) x=(x, c, y) .
$$

So, generally speaking, $[x, y]^{(c)} \neq 0$.

\section{REFERENCES}

1. K. McCrimmon, Homotopes of alternative algebras, Math. Ann. 191 (1971), 253-262. MR 47:1899

2. R. Schafer, Alternative algebras over an arbitrary field, Bull. Amer. Math. Soc. 49 (1943), 549-555. MR 5:33d

3. K. A. Zhevlakov, A. M. Slin'ko, I. P. Shestakov, and A. I. Shirshov, Rings that are nearly associative, Academic Press, New York and London, 1982. MR 83c:17001

Department of Mathematics, Ohio State University, Columbus, Ohio 43202

E-mail address: brkvch@math.ohio-state.edu 